\title{
Figuras interaccionales y propósito comunicativo en conversaciones coloquiales, entrevistas periodísticas y entrevistas del proyecto PRESEEA
}

\author{
Amparo García-Ramón ${ }^{1}$
}

Recibido: 7 de junio de 2017 / Aceptado: 3 de abril de 2020

Resumen. Este trabajo explora la creciente conversacionalización de los discursos mediáticos a partir de un análisis contrastivo del género entrevista frente a dos géneros vecinos: la conversación coloquial y la entrevista sociolingüística. Para ello, en primer lugar, se comparan los patrones interaccionales de los tres géneros mediante una herramienta de visualización. Los resultados muestran que la entrevista periodística es más cercana a la conversación que la sociolingüística. En segundo lugar, se argumenta que algunas de las diferencias estructurales observadas pueden explicarse a partir del propósito comunicativo. Las particularidades del género conversación se relacionan con la ausencia de propósito transaccional. El contraste entre ambos tipos de entrevista se relaciona con una diferencia en el tipo de transacción informativa: mientras que en la entrevista sociolingüística interesa la producción de formas lingüísticas, en la periodística se negocia el acuerdo, lo cual repercute en la estructura interaccional.

Palabras clave: entrevista; figuras interaccionales; propósito comunicativo

\section{[en] Interactional figures and communicative purpose in spontaneous conversations, journalistic interviews and sociolinguistic interviews}

\begin{abstract}
This study explores the conversationalisation of media discourse through a contrsative analysis of journalistic interviews and two related genres, spontaneous conversations and sociolinguistic interviews. To do so, in the first place, interactional patterns in the three genres are compared using a visualization tool. The results show that journalistic interviews are closer to conversation than sociolinguistic interviews. Secondly, it is argued that some of the structural differences can be explained by taking into account the communicative purpose of each genre. The distinctive features of conversation are related to the absence of a transactional purpose. The contrast between both types of interview is related to a difference in the type of informative transaction: while in sociolinguistic interviews it is only the production of linguistic forms that is important, in journalistic interviews agreement is negotiated; this has consequences on the interactional structure.
\end{abstract}

Keywords: interview; interactional figures; communicative purpose

Cómo citar: García-Ramón, Amparo (2020). Figuras interaccionales y propósito comunicativo en conversaciones coloquiales, entrevistas periodísticas y entrevistas del proyecto PRESEEA. Círculo de Lingüística Aplicada a la Comunicación 82, 119-136, http://dx.doi.org/10.5209/clac.68968

Índice. 1.Introducción. 2. Conversación frente a entrevista: estudios previos sobre sus características estructurales y su propósito comunicativo. 2.1. Características estructurales. 2.2. Propósito comunicativo. 3. Metodología. 3.1. Corpus. 3.2. La aplicación de los dientes de sierra. 4. Variedad y frecuencia de figuras interaccionales en conversaciones coloquiales, entrevistas periodísticas y entrevistas sociolingüísticas. 5. Relaciones entre la distribución de figuras interaccionales y el propósito comunicativo. 5.1. Conversación frente a entrevista. 5.2. Entrevista periodística frente a entrevista sociolingüística. 6. Conclusión. Bibliografía

\section{Introducción}

En las últimas décadas se ha puesto de manifiesto una tendencia hacia la "coloquialización" (Briz, 2013a; Narbona, 2009), "conversacionalización" (Clayman y Heritage, 2002; Fairclough, 1995) o "inmediatización" (López Serena, 2014) de los discursos públicos, la cual implica que se plantee, incluso, la pertinencia de utilizar ciertos discursos mediáticos para el estudio del español coloquial (López Serena, 2008).

${ }^{1}$ Grupo Val.Es.Co, Universitat de València. Correo electrónico: Amparo.Garcia-Ramon@uv.es 
Concretamente con respecto al género entrevista, uno de los aspectos más notables de esta creciente coloquialización es que, frente a entrevistas tradicionales en que primaba la neutralidad (Clayman, 1988), se da hoy una mayor implicación de ambos hablantes en su discurso, la cual es más propia de situaciones de inmediatez comunicativa. Ello se traduce en la presencia de rasgos lingüísticos característicos de la conversación, e.g., interrupciones de ambos hablantes (Fairclough, 1995) o, por parte del entrevistador, intervenciones fático-valorativas (Delin, 2000) o exposición de opiniones (Cuenca, 2013). Este trabajo analiza el género entrevista periodística (EP), frente a dos géneros vecinos, la conversación coloquial (CC) y la entrevista sociolingüística (ES). Se persiguen dos objetivos interrelacionados.

El primer objetivo es comparar la estructura interaccional en los tres géneros. Para ello, se emplea una herramienta de visualización llamada "dientes de sierra" (en adelante, DS) presentada en Briz (2013), ampliada en Espinosa-Guerri (2016) y aplicada en Espinosa-Guerri y García-Ramón (2019) mediante la cual, a partir de la transcripción previa del audio, se señalan gráficamente las relaciones entre cada intervención y aquellas intervenciones a las cuales reacciona o las cuales provoca. Cuando se aplica este sistema a interacciones completas, se obtiene una representación gráfica esquemática de toda la interacción, sin residuos. En un trabajo anterior (Espinosa-Guerri y García-Ramón, 2019), se propuso el término figura para referirse a cada uno de los dibujos diferenciables que se generan a partir de los diferentes tipos de agrupamientos de intervenciones, dependiendo de las relaciones de inicio y reacción existentes entre ellas. El segundo objetivo es mostrar que los resultados del primer objetivo se pueden explicar, al menos en parte, a partir del propósito comunicativo de cada género.

Con respecto al primer objetivo, los resultados muestran que se produce una gradación en lo que respecta a la variedad de figuras. La CC es el género que presenta mayor variedad de figuras, seguido de la EP. Esta gradación se mantiene también en lo que respecta a la distribución de figuras según su frecuencia. Se observa una preferencia en la ES por las figuras lineales, frente a las no lineales, que ganan la importancia en la EP y la CC, y las inconexas, que cobran importancia solamente en la CC. Por tanto, la gradación se puede explicar a partir de dos contrastes relevantes: uno, entre la CC y ambos tipos de entrevista; otro, entre la EP y la ES.

En cuanto al segundo objetivo, se mostrará cómo, en el caso de la CC, la ausencia de un propósito transaccional concreto permite que el orden sea más laxo y, por tanto, existen ocasiones en las que se pierde, incluso, el flujo comunicativo: ello da lugar a la presencia de figuras inconexas que no se dan en ningún tipo de entrevista. En el caso de las entrevistas, existe un propósito transaccional y, más específicamente, informativo: obtener una información de una de las partes en un tiempo limitado, lo cual hace que no puedan aparecer, en principio, figuras inconexas. En cuanto al contraste entre las EP y las ES, se da una diferencia esencial en el tipo de información requerida: mientras que en la ES interesa solamente la producción de tipos textuales (narración, argumentación, etc.) por parte de uno de los hablantes para el análisis de su producción de formas lingüísticas, en la EP interesa la creación conjunta de contenidos y, por tanto, existe mayor implicación de ambos hablantes en su discurso. Ello causa que se produzca en la EP negociación del acuerdo, lo cual repercute en la distribución de figuras interaccionales más variadas y con más frecuencia no lineales en la EP que en la ES. Ambos rasgos, variedad y no linealidad, acercan la EP a la CC y la alejan de la ES.

\section{Conversación frente a entrevista: estudios previos sobre sus características estructurales y su propósito comunicativo}

Cabe señalar en primer lugar que, aunque nos referimos en este apartado al género entrevista en un sentido suficientemente abstracto como para englobar ambos tipos estudiados en este trabajo, las caracterizaciones lingüísticas del género entrevista han tendido a centrarse en la entrevista periodística. Existen trabajos sobre la entrevista sociolingüística (e.g., Fernández Sanmartín et al., 2008; Moreno Fernández, 2011), pero generalmente su objeto de estudio no es la descripción del género en sí mismo, como producto textual; con frecuencia, se trata de reflexiones sobre el proceso de recogida de datos, a partir de los cuales, de forma indirecta, podemos llegar a intuir una definición del género. Dos excepciones son Albelda (2004) y Briz (2010), que parten del modelo Val.Es.Co. de variedades diafásicas para analizar la entrevista semidirigida sociolingüística.

\subsection{Características estructurales}

En la conversación existe una presión constante hacia la minimización del tamaño de los turnos (Sacks, Schegloff y Jefferson, 1974). En la entrevista, en teoría, los entrevistados hablan de manera extendida, mientras que los entrevistadores tratan de no realizar ninguna aportación que pueda suponer una interferencia (Heritage y Greatbatch, 1991: 102). De hecho, varios estudios demuestran que los entrevistadores ni siquiera ofrecen intervenciones fáticas (backchannelling) ("mhm", "yeah") a los entrevistados (Delin, 2000; Heritage, 
2004; Heritage y Greatbatch, 1991). En la misma línea, Delin (2000: 93-94) explica que en la entrevista aparecen silencios entre unas intervenciones y otras: esto es porque los entrevistadores no solamente escuchan la intervención de los entrevistados sin interrumpir, sino que esperan brevemente antes de la próxima pregunta para confirmar que, en efecto, el entrevistado ha completado su intervención. Contrariamente a este esquema, en la conversación, los turnos largos pueden llegar a tomarse como una imposición del hablante; por tanto, es necesario que el oyente produzca intervenciones colaborativas y, por la misma razón, se dan más solapamientos e interrupciones.

Varias caracterizaciones del género entrevista apuntan, por una parte, a la existencia de un patrón básico estricto y, por otra, a la relativización de ese patrón. Se dice de la entrevista que posee una estructura basada idealmente en el par adyacente pregunta-respuesta (Briz, 2010b; Méndez, 2003) y, más concretamente, en secuencias de pregunta breve y respuesta larga (Cuenca, 2013). La entrevista y el debate son géneros "no tan libres en la conducta interaccional" como la conversación cotidiana (Briz, 2010b: 129). Predominan, por tanto, las intervenciones solamente iniciativas y solamente reactivas, frente a las intervenciones reactivoiniciativas, más propias de la conversación (Ibid.). De hecho, se podría decir que la violación sostenida de la estructura pregunta-respuesta transforma la entrevista en una conversación (Cuenca, 2013).

Para algunos autores, independientemente de las expectativas del género, las entrevistas "ganan en interés periodístico o televisivo en la medida en que la interacción alcanza un alto grado de dialogismo (...), más allá de los guiones previamente pactados y de la sucesión mecánica de preguntas más o menos breves y largas respuestas" (Salvador y Marín, 2013: 330). Esta idea entronca con el análisis de Clayman (2004) sobre la evolución de patrones interrogativos, cada vez menos prototípicos, en los géneros televisivos. Según el autor, en las últimas décadas, la interacción se ha situado en un lugar de privilegio dentro de la esfera pública. Debido a ello, tanto los periodistas como los representantes públicos -especialmente, los políticos- están obligados a demostrar una elevada competencia conversacional. Para los periodistas, la capacidad conversacional puede considerarse hoy incluso más relevante que la capacidad para recabar y analizar datos, más valorada en el modelo anterior. Un ejemplo paradigmático de esta tendencia, para el español, es la entrevista política de Ana Pastor analizada por Cuenca (2013): de las 120 intervenciones de la periodista, solamente 55 son preguntas, el resto son opiniones y valoraciones.

\subsection{Propósito comunicativo}

Parece existir consenso sobre la idea de que el propósito de la conversación es interpersonal (Briz, 1998). Se puede defender incluso que la conversación carece de un propósito propiamente dicho (Searle, 1992). Los géneros institucionales, en cambio, están centrados en la consecución de una tarea (Heritage y Greatbatch, 1991). En el caso de la entrevista, por tanto, el propósito es transaccional (e.g., Briz, 1998; Méndez, 2003; Pilleux, 1995), aunque la naturaleza de esa transacción no es tan evidente.

Se ha señalado que existen varios tipos de transacción posibles. Méndez (2003: 198) propone que, por una parte, se produce en la entrevista periodística una especie de compra-venta de intereses entre los interlocutores, en términos de imagen (yo proyecto tu imagen de buen periodista, tú proyectas la imagen que quiero dar de mí a la sociedad). Por otra parte, la entrevista es un diálogo para un público externo (Heritage y Greatbatch, 1991) y, por tanto, se da otro tipo de transacción entre los productores de la entrevista y el público, es decir, una finalidad global de la entrevista, como producto acabado. En este trabajo, nos centramos en este segundo nivel de transaccionalidad.

La finalidad global de la entrevista es esencialmente informativa: para Heritage (1985), se trata de un lugar privilegiado para la creación de contenido informativo. Méndez (2003: 201) explica que la finalidad global que se persigue es el "máximo rendimiento informativo". El objetivo es "transmitir información acerca de lo que el entrevistado piensa, sabe, ha hecho o hará" a un público. Todas las intervenciones del entrevistador se dirigen a ese propósito, mientras el entrevistado trata de adaptarse a ello.

Como se señalaba anteriormente, en los trabajos sobre la entrevista sociolingüística, el objeto de estudio suele ser la revisión de las técnicas de grabación para discutir cuál es la forma más eficiente de llegar al fin transaccional, especialmente relacionados con la minimización de la paradoja del observador (Labov, 1972). Por tanto, lo que sí está muy explicitado en estos estudios es precisamente el propósito: el género es una construcción artificial (Cabedo, 2009), planificada para llegar al idiolecto del entrevistado y, en lo agregado, describir el conjunto de una enorme cantidad de idiolectos, para llegar a la reconstrucción de los dialectos correspondientes (sociales, geográficos, etc.). 


\section{Metodología}

\subsection{Corpus}

El corpus analizado contiene un total de 76.983 palabras (2.368 intervenciones) y está compuesto por diez interacciones diádicas: cuatro CC, tres EP y tres ES. Se han seleccionado interacciones de solo dos participantes para poder contrastar conversaciones y entrevistas. Se ha querido con ello minimizar la posibilidad de que, si aparecen más figuras en conversación, se deba a la mayor cantidad de hablantes.

Las cuatro CC $(0011,0031,0033$ y 0039) pertenecen al corpus Val.Es.Co. 2.0 (Cabedo y Pons, en línea). Las EP, emitidas en televisión y transcritas para un trabajo anterior (García-Ramón, 2018), tienen como entrevistados a políticos en activo (Esperanza Aguirre, Pablo Iglesias y Pedro Sánchez). En ellas aparecen tres entrevistadores diferentes (Jordi Évole, Ana Pastor y Risto Mejide, respectivamente). Las entrevistas sociolingüísticas pertenecen al corpus del PRESEEA (Proyecto para el Estudio Sociolingüístico del Español de España y de América). Se han seleccionado grabaciones de tres zonas diferentes: Alcalá (ALCA_H23_007), Madrid (MADR_M33_054) y Valencia (VALE_M33_010).

\subsection{La aplicación de los dientes de sierra}

La herramienta de visualización DS (Briz, 2013; Espinosa-Guerri, 2016; Espinosa-Guerri y García-Ramón, 2019) parte del sistema de unidades Val.Es.Co. (Briz y Grupo Val.Es.Co., 2003; Grupo Val.Es.Co., 2014), especialmente, de la distinción entre intervención iniciativa (que genera acción conversacional posterior), intervención reactiva (que reacciona a un segmento de habla anterior) e intervención reactivo-iniciativa (que reacciona a un segmento lingüístico y genera una reacción lingüística). Mediante los DS se señalan gráficamente las relaciones existentes entre cada intervención y aquellas intervenciones a las cuales reacciona y aquellas intervenciones que genera. Mediante seis convenciones gráficas, se puede aplicar el sistema a cualquier interacción (Imagen 1). En este apartado, resumimos brevemente la metodología de aplicación de la herramienta (para una justificación más detallada de los criterios de aplicación, ver Espinosa-Guerri (2016)).

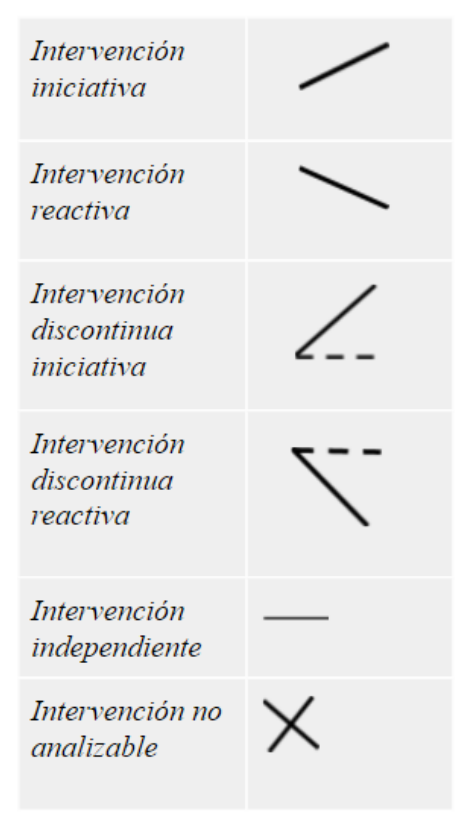

Imagen 1. Convenciones gráficas para la aplicación de los DS

Las líneas unidas con las intervenciones de cada hablante reciben un color específico: en este trabajo, para los casos de entrevista, el entrevistador recibe el color negro y el entrevistado o informante, el color gris. En las conversaciones, el primer hablante se representa mediante el color negro y, el segundo, el color gris.

Las intervenciones iniciativas se señalan mediante una línea diagonal y las reactivas que responden a ellas, mediante una línea diagonal inversa. Así, dos intervenciones, una iniciativa y una reactiva, quedarán unidas como se muestra en (1).

$$
<\begin{aligned}
& \text { E: ¿cuántos años tiene? } \\
& \text { I: setenta y cinco // }
\end{aligned}
$$


Cuando se dan varias intervenciones encadenadas de carácter reactivo-iniciativo, se produce un dibujo en forma de zigzag (2). Este es el molde idealizado de una interacción conversacional y también la situación por defecto, la que se da cuando no está ocurriendo nada reseñable desde el punto de vista estructural.

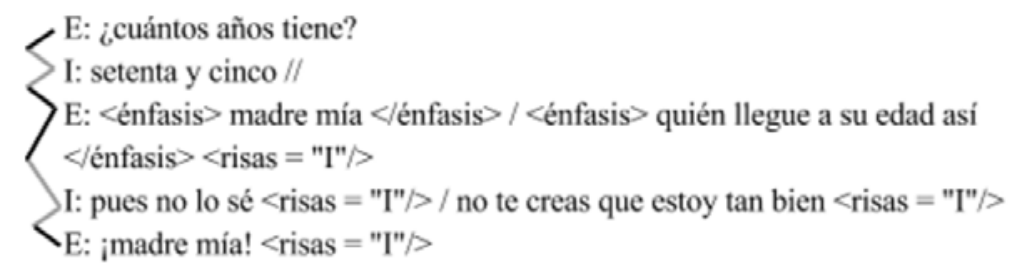

(PRESEEA MADR_M33_054)

Una intervención discontinua, dentro de la cual se intercalan breves intervenciones colaborativas o intentos fallidos de interrupción, se señala añadiendo al esquema básico una línea de puntos cuando finaliza la intervención discontinua, como se muestra en (3). Si la intervención discontinua es reactiva, se invierte el dibujo, es decir, se coloca la línea de puntos en la parte superior de la intervención discontinua. Las intervenciones de E (“isí?”, “¡no me lo puedo creer!”, “y al agua” y "uhum”) son contribuciones colaborativas que no constituyen turno puesto que no obtienen aceptación social (Briz y Grupo Val.Es.Co., 2003; Grupo Val.Es.Co., 2014). Como son únicamente reactivas (no reactivo-iniciativas) están conectadas únicamente con la intervención inicial de I (no con las posteriores).

(3)

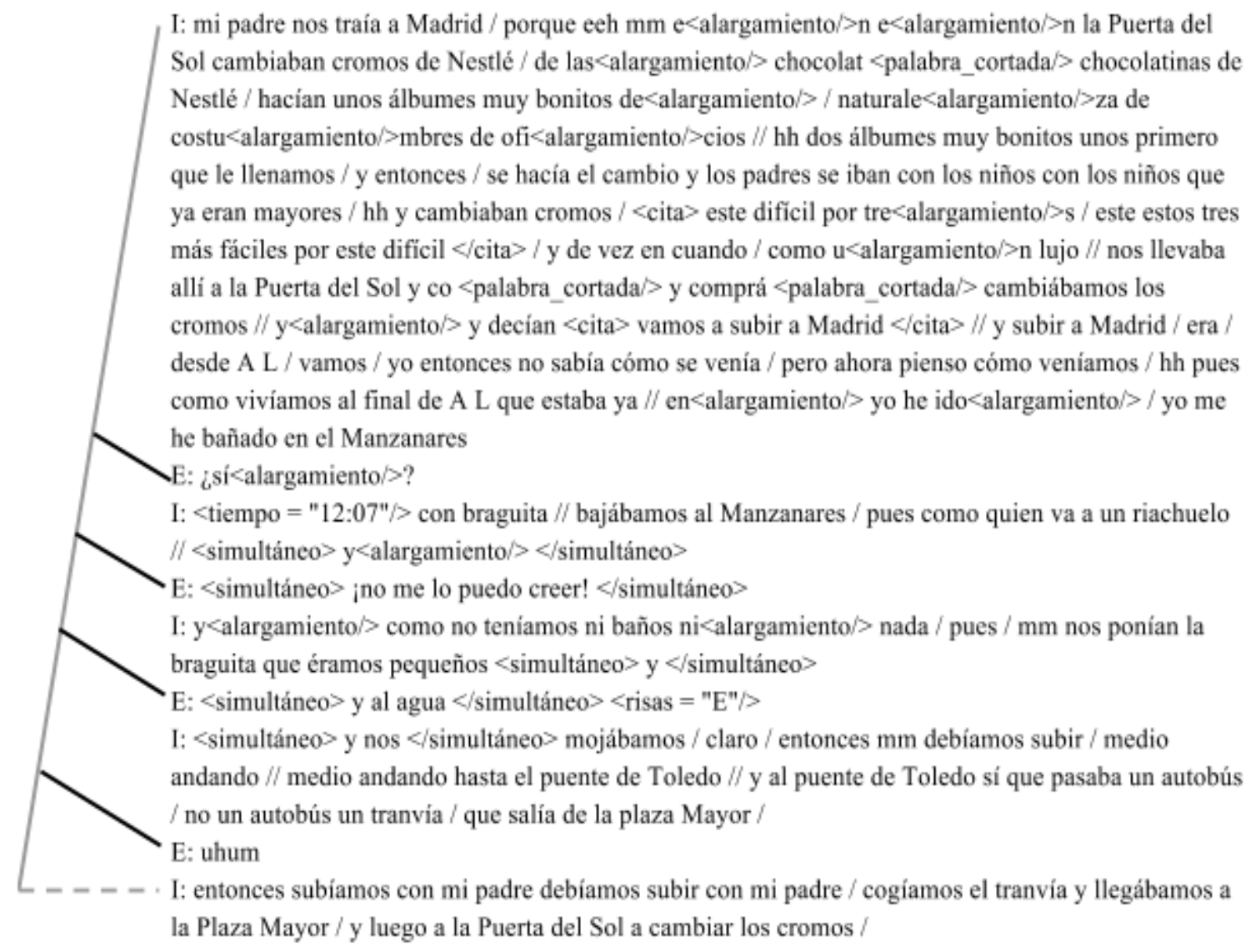

(PRESEEA MADR_M33_054)

Cuando existe un par adyacente o intercambio marco superordinado, dentro del cual se insertan varios tipos de digresión, las digresiones pueden tener diversos grados de engarce con el intercambio marco. Ello se señala uniendo el segmento digresivo tanto con la intervención iniciativa del marco como con la reactiva (4), solamente con la intervención iniciativa (5) o con ninguna de ellas (en (6), el intercambio formado por “¿quieres media?” y "mm ¡ay! sí $\downarrow$ vale”). 
(4)

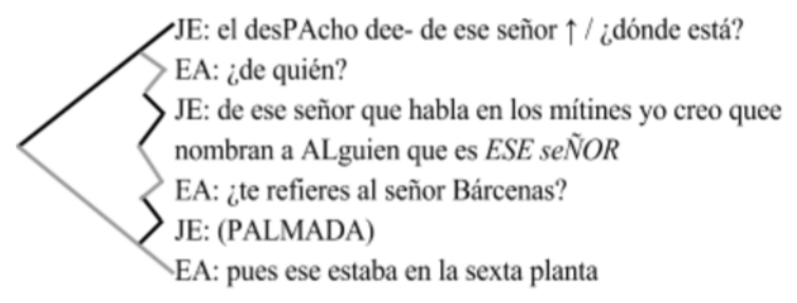

(Entrevista de J. Évole a E. Aguirre)

(5)

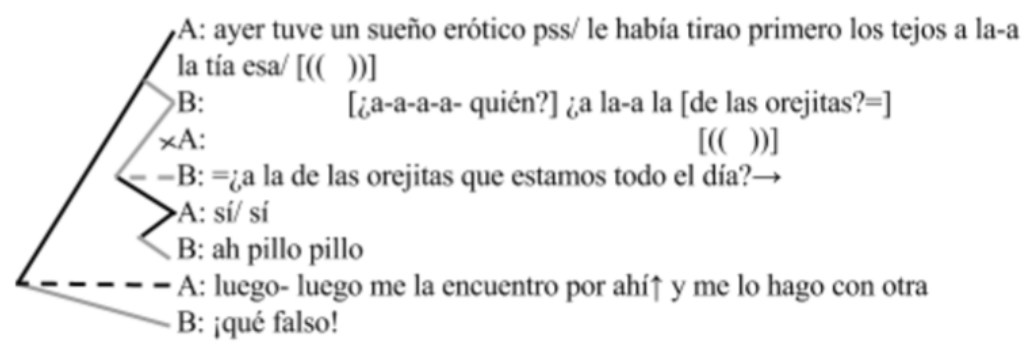

(Val.Es.Co. 2.0, Conversación 0011)

(6)

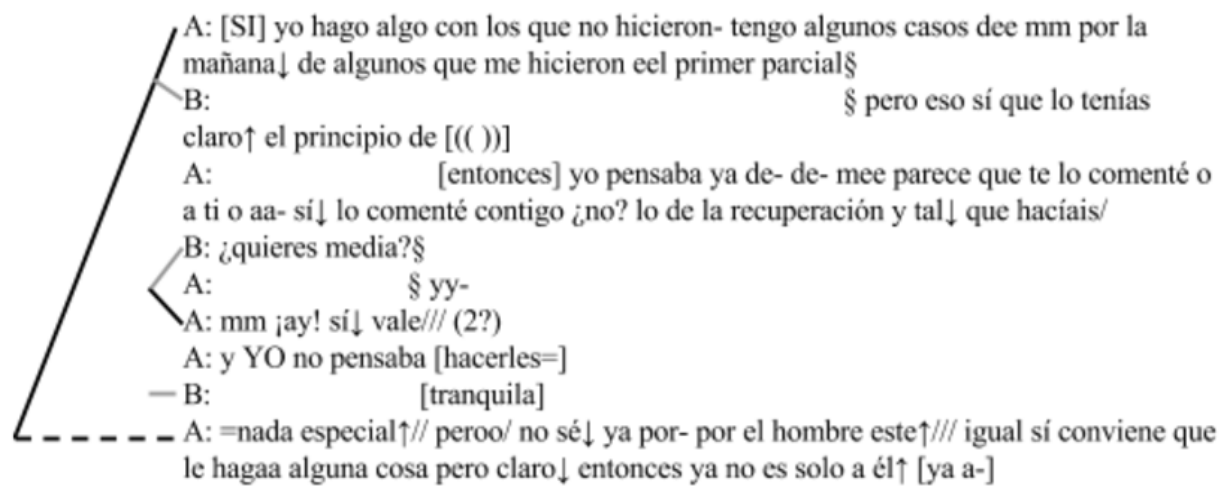

(Val.Es.Co. 2.0, Conversación 0031)

Una intervención independiente, que no reacciona a ningún otro segmento y que tampoco provoca ninguna reacción verbal, se señala mediante una raya horizontal ("tranquila" en (6)). Por último, las intervenciones que no pueden ser analizadas debido a la baja calidad del audio o a que no se dispone de suficiente contexto se señalan mediante una $x$ (la intervención ininteligible de A en (5)). Cada una de las muestras del corpus ha sido analizada por dos investigadoras de manera independiente. Posteriormente, ambos análisis se han sometido a una nueva evaluación conjunta para establecer un análisis definitivo.

\section{Variedad y frecuencia de figuras interaccionales en conversaciones coloquiales, entrevistas periodísticas y entrevistas sociolingüísticas}

La aplicación sistemática de los DS permite observar varios dibujos generados por los tipos de agrupamientos entre intervenciones o figuras conversacionales que presentábamos en un estudio anterior (Espinosa-Guerri y García-Ramón, 2019) y reproducimos a continuación (Imagen 2). Las figuras tienen nombres basados en similitudes visuales para facilitar la referencia a cada una de ellas. Cada intervención se adscribe a una figura. Hay intervenciones que están en la frontera entre dos figuras y, por tanto, cuentan como parte de ambas figuras. Como se explica en ese trabajo, la tipología es provisional porque se basa en el análisis de un corpus reducido en tres sentidos: en primer lugar, solamente se analizan interacciones de dos hablantes; en segundo lugar, solamente se analizan tres géneros (conversaciones, entrevistas periodísticas y entrevistas sociolingüísticas); en tercer lugar, se analiza solamente una selección pequeña de muestras de cada uno de ellos. Cabe esperar que se encuentren otras figuras si se amplía el corpus para incluir interacciones de más hablantes, otros géneros o más muestras de los géneros analizados hasta el momento. 
Sin embargo, la tipología es exhaustiva en el sentido de que agota todas las posibilidades del corpus analizado.

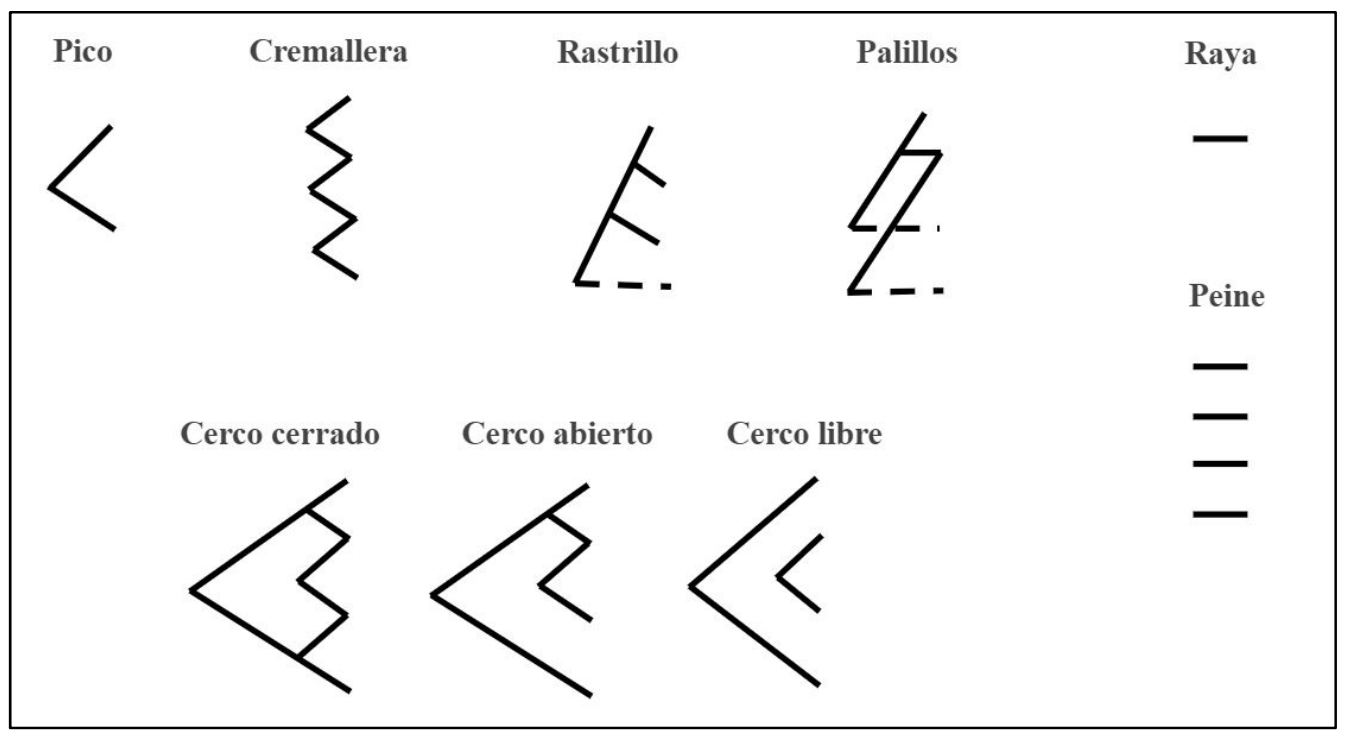

Imagen 2. Tipología provisional de figuras interaccionales (Espinosa-Guerri y García-Ramón, 2019)

La distribución de figuras en cada género se muestra a continuación (Tabla 1). El orden de las figuras se ha establecido a partir de su frecuencia en la CC. Los porcentajes se refieren a la proporción de intervenciones, del total de intervenciones dentro de cada género, que corresponden a cada figura.

\begin{tabular}{|c|c|c|c|c|}
\hline Nombre de la figura & Representación gráfica & $\mathrm{CC}$ & EP & ES \\
\hline Cremallera & & $40,46 \%$ & $44,50 \%$ & $55,74 \%$ \\
\hline Rastrillo & & $37,96 \%$ & $30,70 \%$ & $33,02 \%$ \\
\hline Palillos & & $8,79 \%$ & $11,58 \%$ & $1,87 \%$ \\
\hline Cerco abierto & & $5,19 \%$ & $10,33 \%$ & $3,75 \%$ \\
\hline Cerco cerrado & & $1,80 \%$ & $1,35 \%$ & $0 \%$ \\
\hline Peine & & $1,70 \%$ & $0 \%$ & $0 \%$ \\
\hline Cerco libre & & $1,60 \%$ & $0,58 \%$ & $0 \%$ \\
\hline
\end{tabular}




\begin{tabular}{|l|c|c|c|c|}
\hline Pico & $<$ & $1.60 \%$ & $0.58 \%$ & $5.62 \%$ \\
\hline Raya & - & $1.00 \%$ & $0.10 \%$ & $0 \%$ \\
\hline
\end{tabular}

Tabla 1. Resultados: presencia de figuras interaccionales en CC, EP y ES

El único género en que aparecen todas las figuras detectadas es la CC. Este resultado es coherente con la idea, repetidamente defendida en la literatura, de que la conversación es el género más abarcador en el sentido de que incluye el abanico más amplio de posibilidades interaccionales (Heritage, 2009). Esta idea, a su vez, ha sido uno de los argumentos empleados para defender que la conversación goza de una cierta primacía frente a los demás géneros dialógicos (Heritage, 2013; Schegloff, 2006). Metodológicamente, ello se traduce en la propuesta del AC de analizar los diferentes tipos de discursos institucionales como géneros marcados a partir de su comparación con la conversación como género no marcado. En la EP, encontramos todas las figuras de la tipología excepto una (peine). La ES supone una reducción considerable de la variedad de figuras (no hay casos de cerco cerrado, peine, cerco libre o raya). Según estos resultados, la ES sería el género más marcado, frente a la $\mathrm{EP}$, que aparece como género intermedio.

En cuanto a la frecuencia de cada figura, se observa, de nuevo, que los resultados de la EP se asemejan más a los de la CC que los de la ES. En los tres géneros, las dos figuras claramente predominantes son la cremallera y el rastrillo. En la CC y la EP, las figuras aparecen en el mismo orden de frecuencia (a excepción del peine, que no aparece en la EP):

cremallera $>$ rastrillo $>$ palillos $>$ cerco abierto $>$ cerco

cerrado $>$ (peine $)>$ cerco libre $>$ pico $>$ raya

El orden para la ES, en cambio, es el siguiente:

$$
\text { cremallera }>\text { rastrillo }>\text { pico }>\text { cerco abierto }>\text { palillos }
$$

En la ES, como género más marcado, cobra importancia el pico, pierden importancia el cerco abierto y los palillos y directamente desaparecen las demás figuras. Se observa un patrón claro: en la ES existe una tendencia a la aparición de figuras que llamaremos lineales — basadas en relaciones de contigüidad o en relaciones en que, si hay una intervención inserta, se trata de una intervención no turno- (cremallera, rastrillo y pico) frente a las no lineales - basadas en relaciones no contiguas, con intervenciones intercaladas entre una intervención y aquella(s) con la(s) que se relaciona - (palillos, cerco abierto, cerco cerrado, cerco libre), que cobran importancia en CC y EP, y las inconexas - formadas por intervenciones que no se relacionan lingüísticamente con otras intervenciones- (peine y raya) cuya presencia es relevante solo en CC.

\section{Relaciones entre la distribución de figuras interaccionales y el propósito comunicativo}

Proponemos que algunas de las diferencias en la estructura interaccional de los géneros se relacionan con diferencias en el propósito comunicativo. Se dan dos contrastes relevantes (conversación frente a entrevista y EP frente a ES) que se explicarán por separado. La sucesión de intervenciones independientes (peine) aparece como fenómeno específico de la CC. Esta diferencia esencial se relaciona con la ausencia de propósito de la conversación, que propicia la aparición de segmentos discursivamente inconexos. En segundo lugar, se explicará el contraste entre EP y ES. Para ello, se presentarán instancias de un tipo de situación que tiende a resolverse de manera diferente en los dos géneros: las situaciones potencialmente controvertidas. Se defenderá que las diferencias responden al diferente grado de implicación de los hablantes en el desarrollo informativo, el cual depende, a su vez, de la naturaleza de la transacción que se produce en cada caso (basada en el contenido, en el caso de la EP, o basada en la forma, en el caso de la ES). 


\subsection{Conversación frente a entrevista}

Como se ha explicado anteriormente, el fin de la conversación es interpersonal (Briz, 1998). Searle (1992) va más allá al defender que, en realidad, la conversación se define precisamente por carecer de propósito. Independientemente de qué postura adoptemos, parece claro que en la conversación espontánea no existe la obligación de llevar a cabo ninguna transacción determinada. La ausencia de un propósito bien definido favorece la presencia de una figura (peine) que solamente se da dentro del corpus en las muestras de este género. Esta diferencia se relaciona asimismo con la deixis extrema que caracteriza la conversación coloquial y que no está presente en las entrevistas, por razones que exploramos en un trabajo aparte (Espinosa-Guerri y García-Ramón, en preparación).

En (7), los interlocutores emiten varias intervenciones consecutivas que no responden a ningún otro segmento de la conversación y tampoco generan reacciones lingüísticas. Por tanto, se trata de intervenciones que no hacen avanzar informativamente el discurso.

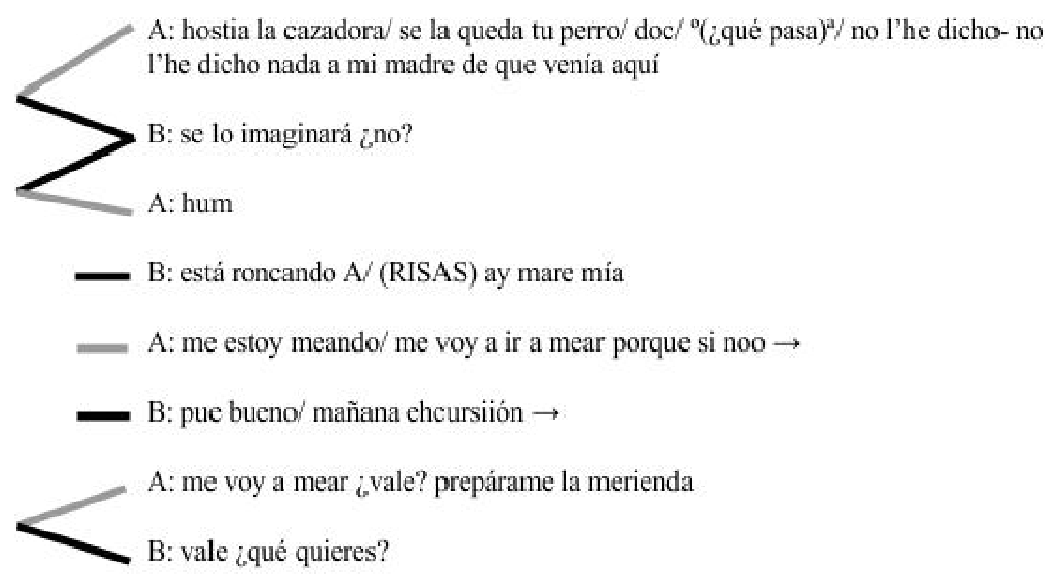

(Val.Es.Co. 2.0, Conversación 0033)

Las entrevistas de cualquier tipo se definen porque en ellas existe un propósito comunicativo bien definido de antemano: obtener una información relativamente específica de una de las partes en un tiempo limitado. La tensión entre la necesidad de obtener la mayor cantidad de información posible y la limitación temporal hace que en las entrevistas el tiempo sea más valioso que en la conversación. Por ello, existe una planificación previa que impide la interrupción del flujo del discurso: los participantes no se pueden permitir desperdiciar tiempo con acciones verbales inconexas, que no obedecen a ningún fin informativo. De hecho, esta necesidad de cumplir con un determinado propósito transaccional se hace explícita en algunos momentos de las entrevistas (8-10) en los cuales alguno de los participantes considera que se están produciendo desviaciones.

En (8), de PRESEEA-Madrid, la informante (I) está hablando de cómo son las chicas en la actualidad y, sin indicación del entrevistador (E), pasa a hablar de su nieta. Ante la sensación de que la entrevista se desvía de su propósito (responder a una serie de preguntas preestablecidas), I propone no entretener más a $\mathrm{E}$ para que pueda cumplir con las directrices ("ihale! no te entretengo pregúntame cosas / que si no no lo vas a rellenar esto ¿eh?"). E desestima esta autocrítica de I porque su función es precisamente lograr que I no perciba la transacción como una entrevista, para que pueda reducir el control sobre su discurso y, de esta forma, producir formas lingüísticas más cercanas a las producidas en situaciones espontáneas.

Asimismo, en (9), de la entrevista de Ana Pastor (AP) a Pablo Iglesias (PI), AP lanza una pregunta que, según su criterio, no está siendo respondida por PI, por lo que le pide que se ciña a la pregunta ("no se me vaya a ese territorio yo le he hecho una pregunta muy directa"). Ante la siguiente respuesta, AP todavía no parece convencida, pero de todos modos da paso a una pregunta nueva, tras hacer referencia a la cantidad de temas que deben tratarse (e, implícitamente, a la carencia de tiempo para detenerse en uno de ellos) ("bueno tengo cientos de preguntas"). 
(8)

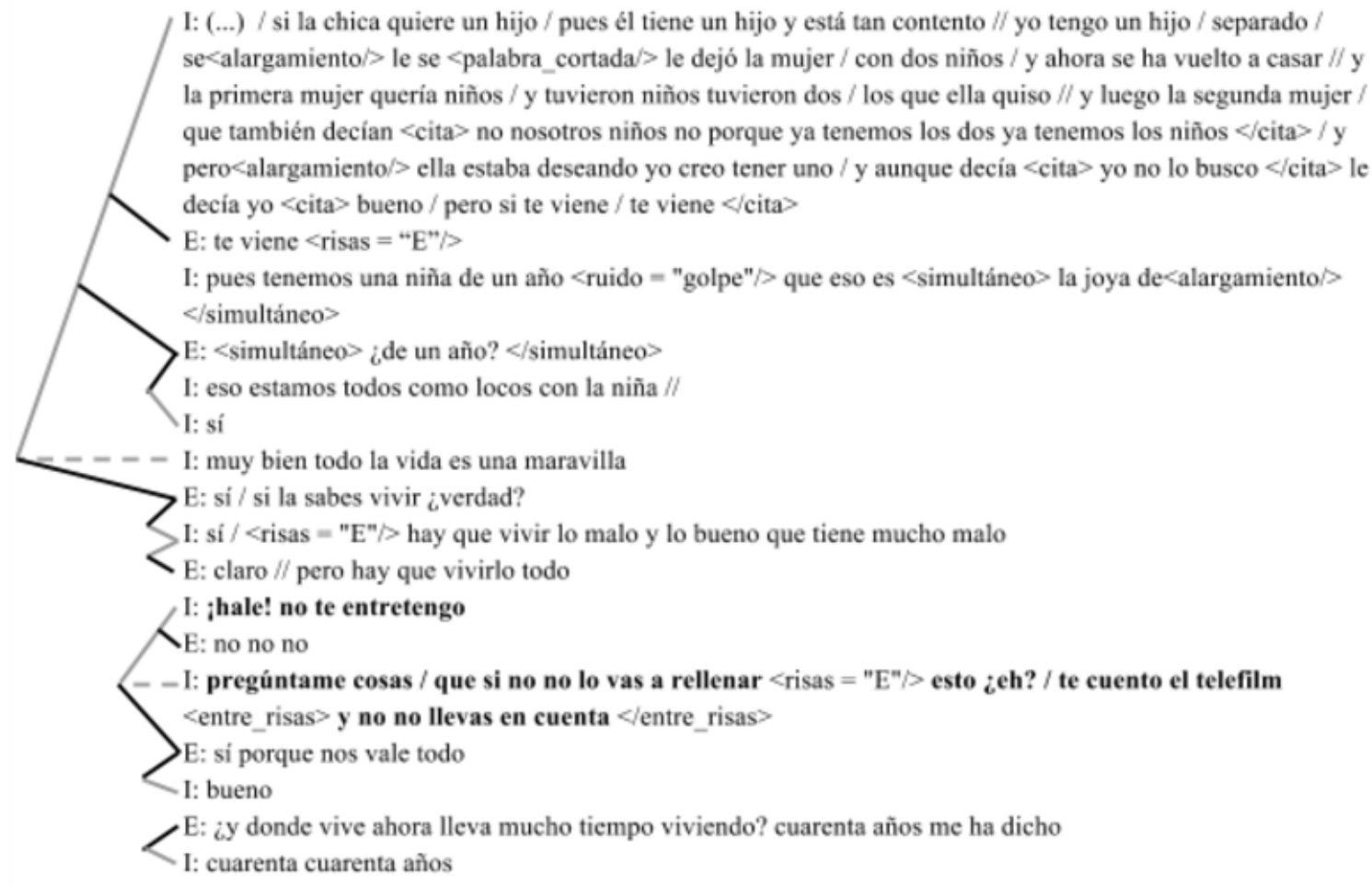

(PRESEEA MADR_M33_054)

(9)

AP: ¿y por qué cree/ que hasta ahora/ no ha habido ningún PAblo Iglesias que haya conseguido quee/

enTIENdan porque usted lo hace como de una manera muy pedagógica y sorprende que vayan a entenderle solo porque usted se lo diga ¿no? [o alguien=]

PI: [por-]

- AP: =tiene que hacerlo

PI: porque nosotros no le debemos nada a nadie/ cuando nosotros hablamos de las puertas giratorias/ estamos diciendo que se está/ secuestrando la democracia cuando alguien por el hecho de haber sido un cargo público termina en consejos de administración de grandes empresas// es como si les compraran/ es decir ahora le pago el sueldo yo a usted/ ahora soy yo el que en última instancia toma las decisiones de lo que usted tiene que hacer/ nosotros deCImos que la corrupción es un sistema que permite/ que gobiernen/ los que no se presentan a las elecciones los que en última instancia ponen a los partidos políticos/maletines con billetes de 500 euros/ los que COMpran a exministros y a expresidentes para sentarles en sus consejos de administración/ nosotroos/ en ese sentido somos insobornables [no le debemos]
AP:
[no se mee-] no se me

PI: nada a nadie

_ AP: no se me vaya no se me VAya a ese territorio yo lo que hecho una pregunta muy directa cómoo va a hacer que los bancos/ que TIEnen viviendas vacías/ se las pueden ceder a quien NO tienen casas y usted me dice prácticamente que les VAN a obedecer/ que van a ir a una reunión y los bancos van a decir/venga a partir de ahora si [_cómo?]

PI: [no no] a nosotros no a la LEY/ nosotros eeh trabajaremos para que haya leyes justas que protejan a los ciudadanos y si HAY en este país alguien que no quiera cumplir las leyes $\uparrow$ no nosotros los jueces de nuestro pais/ les obligarán a cumplir la ley como a cualquier ciudadano§

AP:

§bueno tengo cientos

de preguntas TRES leyes que aprobaría y tres que derogaría para quee/ en este país el paro deje de ser el principal problema para todos

PI: lo fundamenTAL $\uparrow /$ lo fundamental con el tema del paro es hacer lo CONtrario a lo que se ha hecho en este momento//

(Entrevista de A. Pastor a P. Iglesias) 
En (8-9), se comprueba que los participantes de la entrevista mantienen la percepción de estar participando en un evento comunicativo con un propósito transaccional y, concretamente, informativo. En cambio, los participantes de la conversación pueden permitirse interrumpir el flujo informativo. Pueden llegar a producirse, de ese modo, sucesiones de intervenciones inconexas entre sí, únicamente ancladas en el contexto físico de los participantes. Ello favorece la aparición de una figura (peine), que, según lo observado en el corpus, emerge solo en la CC.

\subsection{Entrevista periodística frente a entrevista sociolingüística}

Heritage (1985) proponía que la entrevista es "talk for an overhearing audience". Aunque el autor se refería específicamente a entrevistas periodísticas, esta afirmación es aplicable a todas las entrevistas de nuestro corpus: ninguna de ellas es un evento enteramente privado, puesto que en todas ellas se produce una transacción entre los productores de un texto y sus receptores, los cuales no están físicamente presentes en la interacción. En las EP, la transacción se produce entre los creadores del producto (tanto el entrevistador como los otros agentes implicados en la producción y retransmisión) y el público, interesado en conocer las opiniones y la habilidad argumentativa de un potencial representante político. En las ES, la transacción se produce entre el investigador que realiza y transcribe la entrevista y la comunidad académica interesada en el análisis lingüístico. Esta diferencia en el contexto interactivo general (CIG) (Briz y Albelda, 2013) repercute en cada contexto interactivo concreto (CIC) y ello tiene consecuencias formales, una de las cuales es la diferente distribución de figuras interaccionales.

En este apartado, se analizan situaciones potencialmente controvertidas y se argumenta que estas situaciones tienden a resolverse de manera diferente en la EP y la ES. A partir del análisis de ejemplos concretos trataremos de mostrar que los diferentes modos de resolución en ambos tipos de situación acercan la EP a la CC y la alejan de la ES. Por tanto, aunque este apartado trata de diferenciar la EP de la ES, se emplean fragmentos extraídos del género conversación, que, en la línea de lo sugerido por el AC, se emplea como molde no marcado. El análisis sugiere que en la EP (como en la CC) existe implicación real de los hablantes y, por tanto, negociación en al menos dos sentidos (del acuerdo y de los temas tratados), mientras que en la ES no existe implicación en la misma medida y, por tanto, no hay (o hay menos) negociación. Como trataremos de demostrar, ello da lugar a una mayor frecuencia de figuras no lineales en la conversación y la EP que en la ES.

En ambos tipos de entrevista, existe un cierto interés por llegar a un acuerdo (al menos, por parte de alguno de los interlocutores), pero establecer el acuerdo en las ES es una tarea más sencilla: el investigador no está tan interesado en los contenidos expresados como en la producción de formas lingüísticas. El interés por llegar a un acuerdo en las ES tiene que ver, por una parte, con la preferencia universal por el acuerdo en la interacción (Clayman, 2002; Sacks, 1987) y, por otra, creemos, con la intención de generar una sensación de seguridad en el informante para minimizar la paradoja del observador (Labov, 1983 [1972]). Podría decirse que sí se dan fragmentos de discusión en la ES, puesto que los entrevistadores propician que los informantes generen segmentos de varios tipos (entre ellos, argumentativos) (Calderón Noguera y Alvarado Castellanos, 2011). Asimismo, los interlocutores "se encuentran implicados voluntariamente y participan en la construcción global del texto" (Gómez Molina, 2005: 26). Sin embargo, creemos que esta co-construcción es solamente superficial: no se co-construyen las conclusiones, sino solamente la estructura propia de la entrevista, puesto que todo se enfoca, en última instancia, a la producción de tipos discursivos concretos y no a la transmisión de ideas. En cambio, en las EP, los participantes establecen conjuntamente los contenidos, los cuales son relevantes para la actividad política del país: la búsqueda del acuerdo, en este caso, requiere que uno de los interlocutores (el político) convenza al otro (el entrevistador) de la aceptabilidad de sus propuestas.

En (10-12) encontramos situaciones potencialmente controvertidas de la ES que se resuelven con figuras lineales. En (10), de PRESEEA-Madrid, el entrevistador (E) pregunta a la informante (I) sobre el cambio climático. I expone su opinión de forma detallada, mientras $\mathrm{E}$ alienta la construcción de esta intervención con elementos fáticos ("uhum", "sí sí sí"). I finaliza su intervención mediante la expresión "yo creo". Limita así el alcance de la afirmación al ámbito del yo y da cabida, así, a la existencia de opiniones diferentes. En una situación donde existiera debate real, lo esperable sería que la intervención de I provocase una aportación más significativa por parte de E. Sin embargo, E sencillamente muestra que la opinión de I ha sido recibida y comprendida ("sí/ puede ser") y, acto seguido, da paso a la siguiente pregunta, sin transición (“¿dónde vive usted?”). 


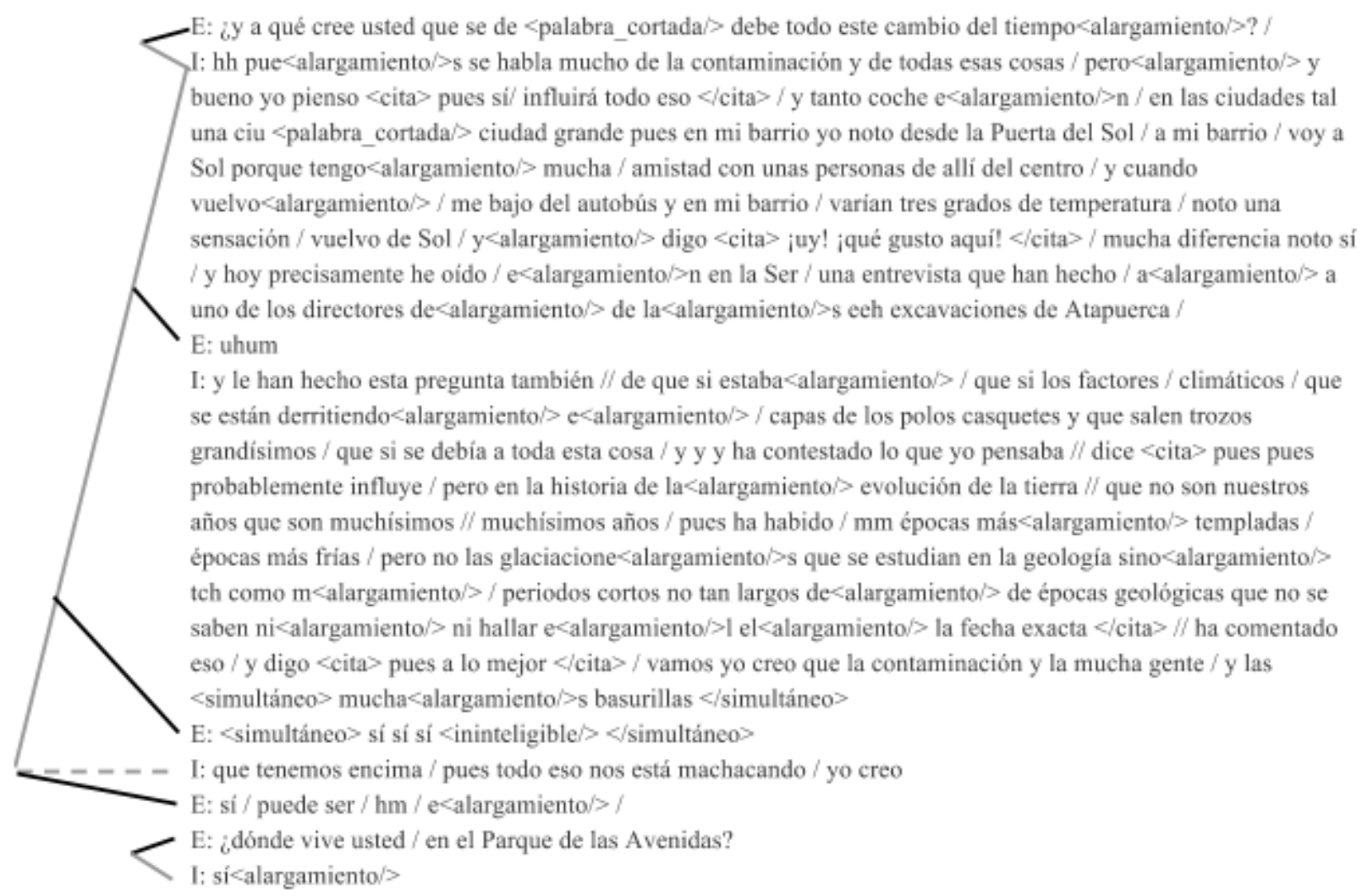

(PRESEEA MADR_033_054)

En (11), de la misma entrevista, I narra su experiencia con las parejas de sus hijos, que se han mostrado reacias a tener hijos. E expresa sorpresa y expone una generalización contraria a esta idea ("normalmente es al revés"). I rechaza la generalización ("no no te creas / ¡qué va qué va! / tch") y ofrece una opinión negativa sobre las mujeres jóvenes en la actualidad ("yo tengo una experiencia muy negativa de las chicas"). La opinión es potencialmente controvertida en tres sentidos: en primer lugar, la percepción mayoritaria es precisamente la contraria a la de E (i.e., que son las mujeres las que tienen más interés por tener hijos); en segundo lugar, E es una mujer más joven que I y, por tanto, el hecho de que I formule una crítica hacia las mujeres jóvenes es una crítica, indirectamente, hacia su interlocutora; en tercer lugar, no existe ninguna razón para que I sea la hablante más autorizada para hablar de las preferencias de las mujeres jóvenes, teniendo en cuenta que E, como mujer joven, vive esa realidad más de cerca. A pesar de que, inicialmente, $\mathrm{E}$ ha formulado la opinión contraria a la de I, a lo largo de la interacción va asumiendo la visión de I como cierta. En la intervención que cierra la cuestión (“¡ah!/ yo siempre vamos hubiera pensado que es al revés / que normalmente son los los hombres los que no quieren no quieren tener hijos") parece que $\mathrm{E}$ se da por informada sobre las preferencias de las mujeres jóvenes. La partícula “¡ah!” parece funcionar aquí como operador de cambio de estado (Heritage, 1984; Vázquez Carranza, 2016): E indica que la aportación de I ha sido informativa y que, por tanto, ha producido un cambio en el conocimiento de E, la cual seguiría manteniendo una visión equivocada si no fuera por esta última aportación. Creemos que, en realidad, E sigue manteniendo su visión inicial, pero la plantea de forma tentativa ("yo siempre vamos hubiera pensado que es al revés") y no se opone, en realidad, a la visión de I, por lo que no se produce una verdadera negociación del contenido propuesto por E.

Una situación similar se da en (12), de PRESEEA-Valencia. El entrevistador (E) pregunta sobre las formas de divertirse que tienen los jóvenes. Ante esa pregunta, la informante (I) produce una larga intervención que incluye una opinión negativa. Hacia el final de su respuesta, I incluye una pregunta confirmatoria (“¿eh?”) y emplea el verbo ver de forma que marca su intervención como una opinión restringida al ámbito del yo ("eso es lo que no / no veo claro"). E simplemente muestra que la contribución ha cumplido con las pautas preestablecidas de la entrevista ("bien Amparo"). Acto seguido, E da paso, sin transición, a la siguiente pregunta (“¿qué prefiere para vivir / la ciudad o el campo?”). Como ocurría en los ejemplos anteriores, la contribución no se somete a debate, como probablemente ocurriría en una situación no marcada (i.e., en una conversación espontánea). 
(11)

～I: <simultáneo> está toda </simultáneo > la gente más lejos // las familias son pequeñitas yo lo veo con mis hijos // a mí me da muchísima pena // tengo dos hijos que no tienen hijos //

$>$ E: $¿$ si<alargamiento $/>$ ?

I: sí // y y porque uno no <alargamiento/> se ha casado // vamos ha vivido con<alargamiento/> chicas tiempo / pues

como si fueran su mujer / pero como las chica $<$ alargamiento/ $>$ s actualmente / eeh son tan feministas que no

quiere $<$ alargamiento $/>$ n problemas de nada $<$ alargamiento $/>/$ mi hijo hubiera tenido hijos $<$ risas = "golpe" $/>/$

pero $<$ alargamiento $>/$ pero las chicas $<$ entre_risas $>$ no han querido nunca $<$ simultáneo $>$ tenerlos $</$ simultáneo $>$

$\langle$ entre_risas $\rangle\langle$ risas $=$ "I" $>$

$\mathrm{E}_{\mathrm{E}:}<$ simultáneo $>$ ¿no han querido? $</$ simultáneo $>/$ normalmente es al revés $<$ risas $=$ "E" $/>$

$>$ I; no no te creas / ¿qué va qué va! / tch

$\mathrm{SE}_{\mathrm{E}: i s i ?}$

$>$ I: $<$ tiempo $=" 22: 20 " />$ yo tengo $<$ entre_risas $>$ una experiencia muy negativa de las chicas $</$ entre_risas $>$

E: $i$ si? $<$ risas $=$ "todos" $/>$

$>$ I: <entre_risas > iqué quieres que te diga! </entre_risas> / yo era mucho más feminista antes que ahora // si /

E: ¡ah! / yo siempre vamos hubiera pensado que es al revés / que normalmente son los los hombres los que que

no quieren no quieren tener hijos quieren $<$ simultáne $0>$ tene $<$ alargamiento $/>\mathrm{r}</$ simultáne $>$

I: <simultáneo> ¡no! </ simultáneo> / ¡uy qué va qué va! / tch ¡qué va!

$>E_{:}: i s i ?$

I: no a lo mejor dicen que no $<$ alargamiento $>/$ tch porque son muy $<$ alargamiento $>$ eeh no toman iniciativas fácilmente

de nada / se lo tiene que dar uno<alargamiento/>

E: ya hecho

I: todo hecho entonces / si la chica quiere un hijo/ pues él tiene un hijo y está tan contento // yo tengo un hijo/ separado / se<alargamiento/> le se <palabra_cortada/> le dejó la mujer / con dos niños / y ahora se ha vuelto a casar //y la primera mujer quería niños / y tuvieron niños tuvieron dos / los que ella quiso // y luego la segunda mujer / que también decian <cita> no nosotros niños no porque ya tenemos los dos ya tenemos los niños $</$ cita $>/ y$ pero $<$ alargamiento $/>$ ella estaba deseando yo creo tener uno $/$ y aunque decia $<$ cita $>$ yo no lo busco $</$ cita $>$ le decía yo $<$ cita $>$ bueno / pero si te viene / te viene $</$ cita $>$

(PRESEEA MADR_033_054)

(12)

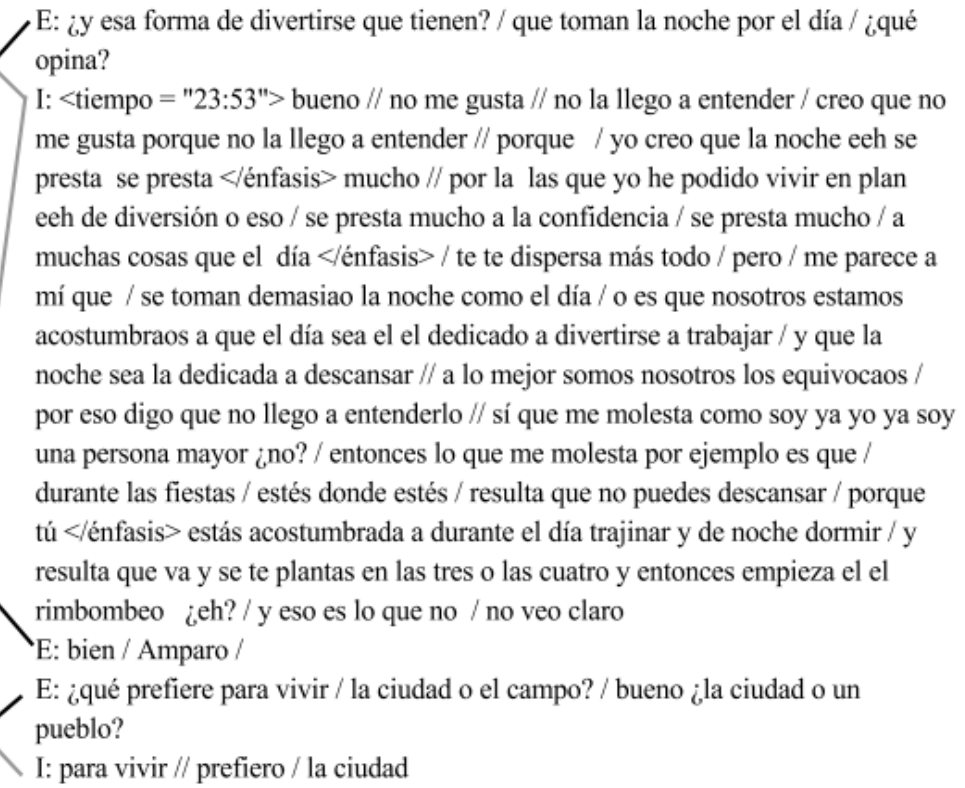

(VALE_M33_010)

En los ejemplos de la ES analizados arriba (10-12), aparecen figuras lineales asociadas con segmentos máximamente ordenados. En el caso de la cremallera, estamos ante una sucesión de intervenciones reactivoiniciativas encadenadas, sin regresiones ni alteraciones: este es el orden por defecto de cualquier interacción $\mathrm{y}$, de hecho, es la figura mayoritaria en todos los géneros. En el caso del rastrillo, uno de los hablantes produce un turno prolongado (frecuentemente una narración o una exposición), mientras que el otro hablante 
produce intervenciones reactivas que no constituyen turno (en el caso de la entrevista, frecuentemente intervenciones colaborativas producidas por el entrevistador), que muestran que el canal está abierto e invitan al hablante que produce la narración o exposición a continuar su turno, sin interrumpir el flujo del discurso.

La argumentatividad de algunos fragmentos de las ES se desarrolla en el plano exclusivamente monológico: no existe una negociación real, con implicación de ambos hablantes, sino, como mucho, de uno de ellos (el informante), que puede llegar a debatir una idea consigo mismo, dentro de su propia intervención. Se producen así segmentos con orden sintáctico propiamente coloquial, con repeticiones y vacilaciones (13), oraciones suspendidas y reinicios (14), autocorrecciones y titubeos (15), etc., pero no figuras interaccionales no lineales, presentes en el discurso conversacional - (13-15) han sido extraídos de (10-12), respectivamente-.

(13)

I: que si se debía a toda esta cosa / y y y ha contestado lo que yo pensaba // dice <cita $>$ pues pues probablemente influye / pero en la historia de la<alargamiento/> evolución de la tierra // (...) pues ha habido / mm épocas más $<$ alargamiento $>>$ templadas / épocas más frías / (...) ha comentado eso / $\mathbf{y}$ digo $<$ cita $>$ pues a lo mejor $</$ cita $>$ / vamos yo creo que la contaminación y la mucha gente

(14)

I: no a lo mejor dicen que no $<$ alargamiento $/>$ / tch porque son muy $<$ alargamiento/ $>$ eeh no toman iniciativas fácilmente de nada

I: yo creo que la noche eeh se presta se presta </énfasis $>$ mucho // por la las que yo he podido vivir en plan eeh de diversión o eso / se presta mucho a la confidencia / se presta mucho / a muchas cosas que el día </énfasis> / te te dispersa más todo / pero / me parece a mí que / se toman demasiao la noche como el día / o es que nosotros estamos acostumbraos a que el día sea el el dedicado a divertirse a trabajar / y que la noche sea la dedicada a descansar // a lo mejor somos nosotros los equivocaos / por eso digo que no llego a entenderlo // sí que me molesta como soy ya yo ya soy una persona mayor ¿no? / entonces lo que me molesta (...)

En los siguientes ejemplos se muestran situaciones controvertidas en las EP (16-17) y en la conversación (18). En los ejemplos de EP, se da una figura no lineal (palillos). La figura se produce debido a la emisión de dos intervenciones discontinuas por parte de dos hablantes diferentes las cuales se desarrollan sin prestar atención a las contribuciones del otro, en paralelo. En (16) la segunda intervención de Ana Pastor (AP) puede leerse de forma ininterrumpida como un discurso completo: "si pero vo- votar era muy fácil también ¿eh? era igual de fácil que inscribirse no había que ir aa ningún sitio hacer clic y punto ¿no?”. Del mismo modo, la intervención de Pablo Iglesias (PI) que responde a la de AP puede leerse como "((votar sí sí)) votar eraa- era sencillísimo pero que hayan votado cien mil personas (...)". La misma figura se da en (17), de la entrevista de Risto Mejide (RM) a Pedro Sánchez (PS). En este fragmento, la intervención de PS se puede leer de forma completa como "¿tú crees que hoy la sociedad catalana está más fracturada? esa es la pregunta” y la de RM se puede leer como "¡no fracturando pero estás preguntando! ¿qué fractura es preguntar? fracturar es decir me voy// eso es fracturar es decir". En todos los casos, la figura termina cuando uno de los interlocutores toma en consideración la última aportación del otro en su una intervención: ello implica que sí existe coconstrucción y que las intervenciones dejan de discurrir en paralelo.

La aparición de esta figura en fragmentos confrontacionales se relaciona con el hecho de que ninguno de los dos participantes quiere renunciar a desarrollar por completo su idea. A diferencia de lo que ocurría en las ES, aquí importa defender el punto de vista propio y ello se logra incluso aunque haya que sacrificar el mantenimiento del orden interaccional recto, convencional o mayoritario representado por las figuras lineales.

Por el mismo motivo, esta figura tiende a aparecer en fragmentos confrontacionales en la conversación coloquial. En (18), dos amigas discuten sobre si es justa o no una manera de obtener un certificado concreto. Al inicio del ejemplo, B comienza a desarrollar una idea ("hombre a mí me da igual que sea") y no termina hasta la última línea (“¿no?”). De forma paralela, A expone una idea contraria. En esta ocasión, la figura aparece invertida porque la intervención de A es solamente reactiva (no produce reacción posterior). 
(16)

AP: esos medios/ privados que usted es tan crítico que luego también recordaremos algún

pasaje glorioso de hemeroteca/ ayer le votaron unas cien mil personas// es MEnos de la

mitad de los que estaban inscritos/ de los simpatizantes como todos conocemos/ ee e ¿eso

que significa? ¿que se han resentido por algo? ¿que no haber incluído a gente como

Echenique le ha podido penalizar? ¿cómo interpreta que MEnos del cincuenta por ciento de

la gente le elija aun siendo más de cien mil personas?

PI: yo creo que es una cantidad MUY alta/ teniendo en cuenta que la inscripción en

Podemos es algo muy sencillo que cualquiera puede hacer en cualquier momento// puede

haber alguien que se inscribiera en Podemos en el mees de agostoo o en el mees dee/ julio

porque tuviera// interés en participar en alguna cosa o porque tuviera/interées en seguir

algún proceso y que en este momento/ digamos eh dice bueno no tengo ganas de participar

en est [ee]

AP: [si] pero vo- votar era muy fácil [también ¿eh? era igual=]

PI:

[((votar sí sí))]

AP: =de fácil que inscribirse no había que ir aa [ningún sitio=]

PI:

[votar eraa-=]

- -AP: =hacer clic y punto ¿no?

- - PI: =era sencillísimo pero que hayan votado cien mil personas en un proceso como este / donde además/ es cierto que/ alGUnos compañeros / con ideas difeRENtes sobre lo que tenía que ser Podemos no han querido/ ni disputar la secretaría general ni disputar el equipo de dirección pues es lógico quee- que MUcha gente dijera / bueno en esta ocasión no quiero participar/aún así más de cien mil personas participando creo que es una / cantidad muy respetable $\S$

AP:

$\S$ yo me refería/ a los otros al más del cincuenta por ciento que no

se ha sumado

(Entrevista de Ana Pastor a Pablo Iglesias)

(17)

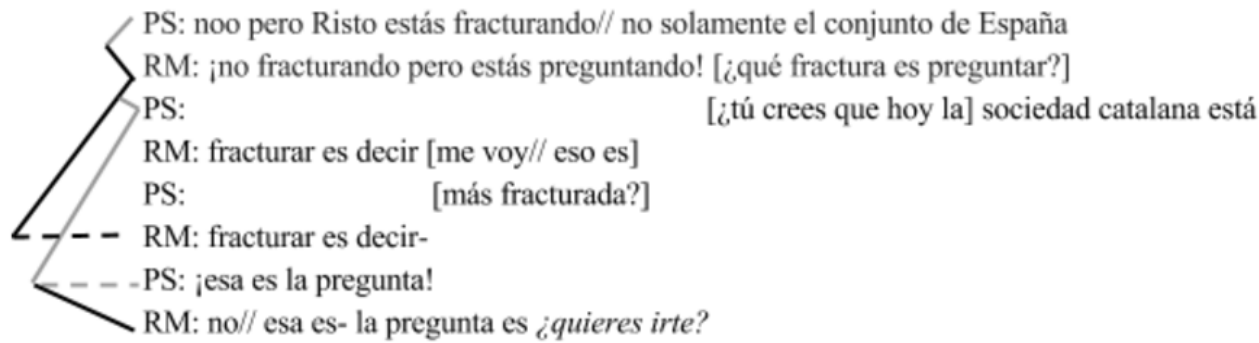

(Entrevista de R. Mejide a P. Sánchez)

(18)

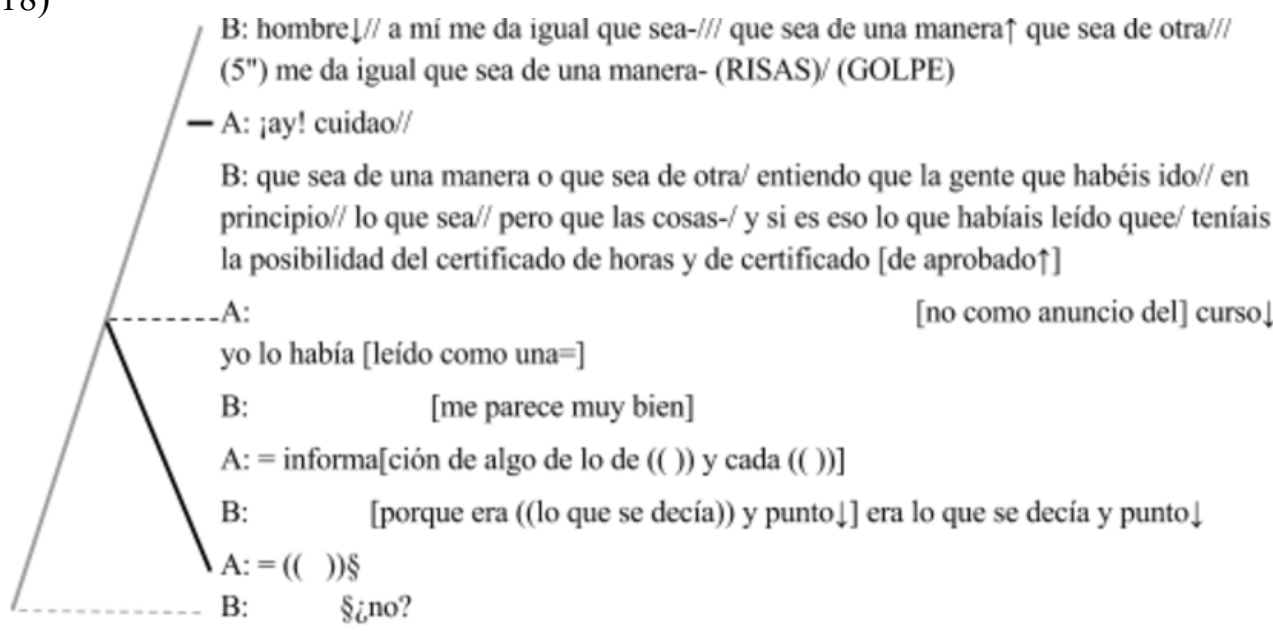

(Corpus Val.Es.Co. 2.0, Conversación 0031) 


\section{Conclusión}

El análisis muestra que existe una gradación en lo que respecta a la variedad de figuras interaccionales (CC > EP $>$ ES) la cual se mantiene, también, en lo relativo a la distribución de esas figuras, según su frecuencia. Dicho de otro modo, tanto en la variedad como en la frecuencia de cada figura, la EP es más cercana a la CC que la ES. Mediante el análisis cualitativo de situaciones interaccionales particulares, hemos tratado de mostrar que la diferente distribución de figuras interaccionales en los tres géneros analizados obedece a diferencias en el propósito comunicativo de cada género. La presencia en la conversación de fragmentos prolongados de intervenciones inconexas se encuentra directamente relacionado con la ausencia de propósito, en contraste con lo que ocurre en ambos tipos de entrevista, en las cuales el propósito está siempre presente para los interlocutores.

Las diferencias entre la EP y la ES se relacionan con el tipo de transacción que se lleva a cabo en cada caso. Una transacción informativa basada en formas lingüísticas (la que se produce en las entrevistas sociolingüísticas) da lugar a patrones conversacionales más lineales, mientras que una transacción basada en el significado (la que se produce en la EP) genera situaciones de negociación del acuerdo y da lugar a patrones conversacionales menos lineales, más propios de la conversación.

La EP comparte con la CC un rasgo esencial: en general, a los interlocutores les importa que las conclusiones alcanzadas sean válidas. Este rasgo es clave porque conlleva que los participantes están implicados en lo que dicen, sea cual sea la consecuencia -mayor en el caso de la entrevista periodística, cuyas repercusiones se sitúan en la esfera de lo público, menor en el caso de la conversación, porque permanece en el ámbito de lo privado-. Tanto en la CC como en la EP, quien calla otorga: ni los periodistas ni los políticos se pueden permitir dejar sin evaluar informaciones que consideren falsas u opiniones que consideren poco plausibles, porque ello equivaldría a conceder su validez. En la ES, el entrevistador otorga por defecto, de manera que se desdibuja el contrato conversacional según el cual permanecer en silencio es dar la razón al otro. Todo esto repercute en la aparición de patrones más o menos variados y más o menos complejos. El análisis presentado más arriba es coherente con esta idea, al mostrar que se produce una gradación (CC $>$ EP $>$ ES) en lo que respecta a la variedad y distribución de figuras según su frecuencia.

El hecho de que en la CC y en la EP tengan una mayor presencia las figuras que hemos llamado no lineales da a estos géneros un carácter en cierto modo más desordenado, lo cual no implica, por supuesto, que se trate de géneros en algún sentido deficientes. Más bien, indica que son discursos genuinamente coconstruidos, a diferencia de lo que ocurre en la ES, género en el cual los discursos solamente se coconstruyen en un nivel superficial y, por tanto, los rasgos estructurales propios de la inmediatez comunicativa se observan solamente en el plano monológico, en las exposiciones de los informantes.

\section{Agradecimientos}

Este trabajo se ha llevado a cabo en el marco del proyecto de investigación Es.Vag.Atenuación: La atenuación pragmática en su variación genérica: géneros discursivos escritos y orales en el español de España y América (MINECO FFI2016-75249-P).

\section{Bibliografía}

Albelda, Marta (2004). Cortesía en diferentes situaciones comunicativas: la conversación coloquial y la entrevista sociológica semiformal. En Bravo, Diana, y Briz, Antonio, eds., Pragmática Sociocultural: estudios sobre el discurso de cortesía en español, 109-134. Barcelona: Ariel.

Briz, Antonio (1998). El español coloquial en la conversación. Esbozo de pragmagramática. Barcelona: Ariel.

Briz, Antonio (2010a). El registro como centro de la variedad situacional. Esbozo de la propuesta del grupo Val.Es.Co. sobre las variedades diafásicas. En Fonte, Irene, y Rodríguez Alfano, Lidia, comps., Perspectivas dialógicas en estudios del lenguaje, 21-56. Nuevo León: Universidad Autónoma de Nuevo León.

Briz, Antonio (2010b). Lo coloquial y lo formal, el eje de la variedad lingüística. En Castañer, Rosa María, y Lagüens, Vicente. eds., De moneda nunca usada. Estudios dedicados a José María Enguita, 125-133. Zaragoza: Institución Fernando el Católico.

Briz, Antonio (2013a). Variación pragmática y coloquialización estratégica. El caso de algunos géneros televisivos (la tertulia). En Fuentes, Catalina, ed., (Des)cortesía para el espectáculo: estudios de pragmática variacionista, 89-125. Madrid: Arco Libros.

Briz, Antonio (2013b). El relato coloquial. Comunicación presentada en el XXVIII Congreso de hispanistas italianos, Pisa, Italia (27-30/11/2013). 
Briz, Antonio, y Albelda, Marta (2013). Una propuesta teórica y metodológica para el análisis de la atenuación lingüística en español y portugués. La base de un proyecto en común (ES.POR.ATENUACIÓN). Onomázein, 28, 288-319. http://roderic.uv.es/handle/10550/33973.

Briz, Antonio, y Grupo Val.Es.Co. (2003) Un sistema de unidades para el estudio del lenguaje coloquial. Oralia, 6, 761.

Cabedo, Adrián (2009). Caracterización prosódica de algunos géneros discursivos. Londres: Lulu.

Cabedo, Adrián, y Pons, Salvador (eds.). Corpus Val.Es.Co 2.0. [http://www.valesco.es]

Calderón Noguera, Donald Freddy, y Alvarado Castellanos, Julia (2011). El papel de la entrevista en la investigación sociolingüística. Cuadernos de Lingüística Hispánica, 17, 1-24. http://www.redalyc.org/pdf/3222/ 322227523002.pdf

Clayman, Steven (1988). Displaying neutrality in television news interviews. Social problems, 35 (4), $474-490$. http://www.sscnet.ucla.edu/soc/faculty/clayman/Site/Publications_files/Clayman\%201988\%20DisplayingNeutrality. pdf

Clayman, Steven (2002). Sequence and Solidarity. In Thye, S.R. y Lawler, E.J., eds., Advances in Group Processes: Group Cohesion, Trust, and Solidarity. Amsterdam: Elsevier Science. Pp. 229-53.

Clayman, Steven (2004). Arenas of interaction in the mediated public sphere. Poetics, 32, $29-49$. http://citeseerx.ist.psu.edu/viewdoc/download;jsessionid=3D15140B384FE3DC7AFDEE9D50F110E3?doi=10.1.1.4 $74.2771 \& \mathrm{rep}=\mathrm{rep} 1 \&$ type $=$ pdf

Clayman, Steven, y Heritage, John (2002). The News Interview. Journalists and Public Figures on the Air. Cambridge: Cambridge University Press.

Cuenca, Maria Josep (2013). Uso del vocativo en la entrevista política: género discursivo y (des)cortesía. Discurso y Sociedad, 7(3), 522-552.

Delin, Judy (2000). The Language of Everyday Life. London: SAGE.

Espinosa-Guerri, Guadalupe (2016). Dientes de sierra: una herramienta para el estudio de la estructura interactiva del discurso dialógico. Normas, 6, 13-27. http://roderic.uv.es/handle/10550/56687

Espinosa-Guerri, Guadalupe, y García-Ramón, Amparo (2019). A preliminary typology of interactional figures based on a tool for visualizing conversational structure. In Loureda, Óscar, Recio, Inés, Nadal, Laura, and Cruz, Adriana, eds., 93-130. Empirical Studies of the Construction of Discourse. Amsterdam: John Benjamins.

Espinosa-Guerri, Guadalupe, y García-Ramón, Amparo (en preparación). Intervenciones independientes, deixis extrema y los límites de la conversación coloquial.

Fairclough, Norman (1995). Critical discourse analysis: the critical study of language. Longman: London/New York.

Fernández Sanmartín, Alba, García Salido, Marcos, Recalde, Motserrat, y Vázquez Rozas, Victoria (2008). Reflexiones metodológicas sobre la técnica de la entrevista semidirigida. Actas del XV Congreso Internacional de ALFAL. Montevideo, Uruguay. https://gramatica.usc.es/ vvazq/pdf_publ/entrevista_semidir_alfal_2008.pdf

García-Ramón, Amparo (2018). Epistemicidad en interacción: (a)simetrías epistémicas en secuencias de acuerdo y su relación con la construcción de roles funcionales en conversaciones y entrevistas. Tesis doctoral. Universitat de València.

Gómez Molina, José Ramón (2005) (coord.). El español hablado de Valencia. Materiales para su estudio. Anejo $\mathrm{n}^{\circ} 58$ de Quaderns de Filologia. Valencia: Universitat de València.

Grupo Val.Es.Co. (2014). Las unidades del discurso oral. La propuesta Val.Es.Co. de segmentación de la conversación (coloquial). Estudios de Lingüística del Español, 35, 13-73. http://www.raco.cat/index.php/Elies/article/viewFile/ $285724 / 373695$

Heritage, John (1984). A Change of State Token and Aspects of Its Sequential Placement. In Atkinson, J. Maxwell, and Heritage, John, eds., Structures of Social Action, 299-245. Cambridge: Cambridge University Press.

Heritage, John (1985). Analyzing News Interviews: Aspects of the Production of Talk for an Overhearing Audience. In van Dijk, Teun, ed., Handbook of Discourse Analysis, Vol.3, Discourse and Dialogue, 95-117. London: Academic Press.

Heritage, John (2004). Conversation Analysis and Institutional Talk. In Sanders, Robert E., and Fitch, Kristine L., eds., Handbook of Language and Social Interaction, 103-146. Mahwah NJ, Erlbaum.

Heritage, John (2009). Conversation Analysis as Social Theory. In Turner, Brian S., ed., The New Blackwell Companion to Social Theory, 300-320. London: Blackwell.

Heritage, John (2013). Language and social institutions: The conversation analytic view. Journal of Foreign Languages, $36 \quad$ (4), 2-27. http://www.sscnet.ucla.edu/soc/faculty/heritage/Site/Publications_files/ Language\%20and\%20Social\%20Institutions.pdf

Heritage, John, and Greatbatch, David (1991). On the Institutional Character of Institutional Talk: The Case of News Interviews. In Boden, Deirdre, and Zimmerman, Donald H., eds., Talk and Social Structure: Studies in Ethnomethodology and Conversation Analysis, 93-137. Cambridge: Polity Press. Labov, William (1972). A Quantitative Study of Sound Change in Progress. Philadelphia: University of Pennsylvania.

López Serena, Araceli (2008). Los medios de comunicación audiovisual como corpus para el estudio de la sintaxis coloquial. En Camacho Taboada, María Victoria, Rodríguez Toro, José Javier, y Santana Marrero, Juana, eds., Estudios de Lengua Española: Descripción, Variación y Uso: Homenaje a Humberto López Morales, 405-437. Madrid: Iberoamericana/Vervuert. 
López Serena, Araceli (2014). De la oralidad fingida a la oralidad simuladora de la realidad. Reflexiones en torno a la coloquialización del discurso como estrategia mediática. Español Actual, 102, 37-75. https://idus.us.es/ xmlui/handle/11441/40123

Méndez García de Paredes, Elena (2003). Lo hablado en lo escrito: la entrevista periodística. Oralia, 6, 147-168.

Moreno Fernández, Francisco (2011). La entrevista sociolingüística. Esquemas de perspectivas. Linred: Lingüística en la Red 9. http://dspace.uah.es/dspace/handle/10017/24166

Narbona, Antonio (2009). Oralidad y escritura, coloquialidad e informalidad. Minervae Baeticae. Boletín de la Real Academia Sevillana de Buenas Letras, XXXVII, 111-119. https://idus.us.es/xmlui/handle/11441/13701

Pilleux, Mauricio (1995). La entrevista como tipo de discurso. Análisis lingüístico. Estudios Filológicos, 30, 95-107.

PRESEEA (2014-). Corpus del Proyecto para el estudio sociolingüístico del español de España y de América. Alcalá de Henares: Universidad de Alcalá. [http://preseea.linguas.net].Consultado: 8/12/2015.

Sacks, Harvey (1987). On the Preferences for Agreement and Contiguity in Sequences in Conversation. In Button, Graham, and Lee, John R.E., eds., Talk and Social Organisation, 54-69. Clevedon: Multilingual Matters.

Sacks, Harvey, Schegloff, Emmanuel, and Jefferson, Gail (1974). A Simplest Systematics for the Organization of TurnTaking for Conversation. Language, 50(4), 696-735.

Salvador, Vicent, y Marín, Maria Josep (2013). Preguntas y preguntas: La gestión de la interacción en la entrevista política. En Soares da Silva, Augusto, Martins, Cândido, Magalhães, Luísa, y Gonçalves, Miguel, eds., Comunicação Política e Económica. Dimensões Cognitivas e Discursivas, 329-347. Braga: Publicações da Faculdade de Filosofia.

Schegloff, Emmanuel (2006). Interaction: The Infrastructure for Social Institutions, the Natural Ecological Niche for Language, and the Arena in which Culture is Enacted. In Levinson, Stephen C., Enfield, Nicholas J., eds., Roots of Human Sociality: Culture, cognition and interaction, 70-96. London: Ber.

Searle, John (1992). Conversation. In Searle, J. et al., (On) Searle on Conversation, 7-30. Amsterdam: John Benjamins.

Vázquez Carranza, Ariel (2016). Aceptación y resistencia: ah y ay como indicadores de cambio de estado. Cuadernos de Lingüística del Colegio de México, 3(2), 71-103. http://cuadernoslinguistica.colmex.mx/index.php/ $\mathrm{cl} /$ article/view/38 